\title{
Study of the structural, thermal, rheological and film properties of functional copolymers of hydroxyethyl acrylate and methyl methacrylate
}

\author{
María Mejia ${ }^{1}$ and Edwin Murillo2*
}

\begin{abstract}
${ }^{1}$ Grupo de Investigaciones Pirometalúrgicas y de Materiales - GIPIMME, Departamento de Ingeniería de Materiales, Universidad de Antioquia - UdeA, Medellín, Colombia

${ }^{2}$ Grupo de Investigación en Materiales Poliméricos - GIMAPOL, Departamento de Química, Universidad Francisco de Paula Santander - UFPS, San José de Cúcuta, Colombia

*edwinalbertomurillo@gmail.com
\end{abstract}

\begin{abstract}
New functional polymers will be prepared using alkyd resins having high solid content (environmentally friendly) and comb-type structural morphology. Different copolymers of hydroxyethyl acrylate and methyl methacrylate (HEMMA) were synthesized by solution polymerization using azo-bis-(isobutyronitrile) (AIBN) as initiator and dimethylformamide as a solvent. The proportions utilized of AIBN were 0.5 (HEMMA1), 1.0 (HEMMA2), 1.5 (HEMMA3) and 2.0 wt. \% (HEMMA4). The conversion percentage of the reaction was higher than $90 \%$. The formation of the copolymers was evidenced by infrared analysis, hydroxyl value, and nuclear magnetic resonance. The intensity of $\mathrm{OH}$ group adsorption increased with the molecular weight and hydroxyl value. The polydispersity index was lower than 1.5. All copolymers exhibited a stable region on viscosity at a shear rate between 0.1 and $10 \mathrm{~s}^{-1}$. The copolymers exhibited good thermal stability, flexibility, and adherence.
\end{abstract}

Keywords: hydroxyethyl acrylate, methyl methacrylate, synthesis, copolymerization, properties.

\section{Introduction}

Acrylic resins have been widely used in the coating and adhesive industries ${ }^{[1,2]}$. These materials have good resistance to ultraviolet light, hydrolysis, oxidation, and its physics drying is fast $\mathrm{t}^{[3]}$. Copolymers obtained from hydrophobic and hydrophilic monomers have a great importance in various applications, such as biocompatible materials, hydrogels, and coatings ${ }^{[4-6]}$. The hydroxyethyl acrylate (HEA) is a soft monomer and is a bi-functional molecule since it presents an $\mathrm{OH}$ group and a double bond ${ }^{[7,8]}$. The glass transition temperature of the polyhydroxyethyl acrylate (PHEA) is estimated at $-15^{\circ} \mathrm{C}^{[7,8]}$. The function of HEA in the copolymers is providing elasticity and the $\mathrm{OH}$ groups ${ }^{[7]}$. MMA is a monofunctional monomer ${ }^{[9]}$ and has been employed in the synthesis of many copolymers ${ }^{[10-12]}$. MMA and HEA can be polymerized by free radical polymerization ${ }^{[8,12]}$. HEA has been employed in some synthesis of polymers ${ }^{[13,14]}$. Reactive monomers (HEA, hydroxyethyl methacrylate, maleic anhydride, etc.) have attracted considerable interest in recent years since they are important precursors for the synthesis of graft copolymers with special properties ${ }^{[5]}$. They can be employed in applications where high molecular weight polymers are not required ${ }^{[15]}$.

PHEA was synthesized without a solvent by free-radical frontal polymerization (FP) at ambient pressure ${ }^{[16]}$. HEA and sodium acrylate (AANa) were grafted on the starch backbone in an aqueous solution to obtain biopolymerbased superabsorbent hydroge ${ }^{[17]}$. Copolymerizations of HEA/methacrylic acid and ethyl acrylate/HEA by free radicals has been conducted in m-xylene employing a temperature range between 70 and $130{ }^{\circ} \mathrm{C}$, and using tertbutyl peroxy benzoate as the initiator ${ }^{[18]}$. HEA/methacrylic acid copolymers with high HEA content were highly crosslinked and found to swell in dimethylsulfoxide (DMSO) ${ }^{[18]}$.

The polymerization of HEA onto chitosan using persulfate as initiator was performed in an aqueous solution. The polymerization rate was much more sensitive to the concentration of the HEA than to the concentration of the initiator $^{[19]}$

Poly(HEA-co-coumaryl acrylate)s were prepared by free radical polymerization using dimethylformamide as solvent and AIBN as an initiator. The copolymers were self-assembled into microspheres in the aqueous phase due to their amphiphilicity ${ }^{[20]}$.

The free radical polymerization is a versatile technique since it presents tolerance to impurities (stabilizers, water, oxygen, etc.), is inexpensive and a wide amount of monomers can be polymerized by this technique ${ }^{[21]}$.

HEMMA copolymers can be used as an alternative to obtaining environmentally friendly alkyd resins (high solid content), but one requirement is that the copolymer have low molecular weight (low viscosity) and also have enough reactive functional groups ( $\mathrm{OH}$ groups) which can react with acid groups of fatty acids. In this work it is intended to obtain functional HEMMA copolymers by solution polymerization employing different proportions of initiator, since this is an important variable to the synthesis of copolymers with low viscosity and adequate properties that in the future allow obtaining of alkyd resins with high 
solid content. The structural, thermal, rheological and film properties of all materials also will be studied.

\section{Experimental Section}

\subsection{Materials}

Sigma-Aldrich supplied HEA, MMA, AIBN, sodium chloride, sodium hydroxide, chlorhidric acid and $\mathrm{N}$, $\mathrm{N}$-dimethylformamide (DMF).

\subsection{Synthesis of the HEMMA copolymers}

The amount of HEA ( $85 \mathrm{~g}$ ) MMA (15 g) and DMF $(200 \mathrm{~g})$ were taken in a reactor. The mixture was heated at $80^{\circ} \mathrm{C}$ and stirred at $100 \mathrm{rpm}$ under a nitrogen atmosphere. In every case, the respective proportion of $\operatorname{AIBN}(0.5,1.0$, 1.5 and $2.0 \mathrm{wt} \%$ with respect to the proportions of HEA and MMA) was added to the reactor and the mixture reacted for $5 \mathrm{~h}$. The solid content in each case was $50 \mathrm{wt} \%$. The reaction conversion related to the formation of HEMMA or HEA and MMA homopolymers was studied by gravimetric analysis and the analysis was conducted in triplicate. To accomplish, the samples were collected at different time intervals during the reaction (these samples were not previously purified) and weighed. A small amount of methyl ethyl hydroquinone $(1 \mathrm{wt} \%)$ was added to these samples to prevent the reaction during solvent evaporation process. These samples were kept in vacuum oven at $50^{\circ} \mathrm{C}$ for $48 \mathrm{~h}$. Finally, the samples were taken out and weighted. It is worth mentioning that samples employed for the determination of the conversion percentage were not used to others analysis but discarded. The copolymers were named HEMMA1 $(0.5 \mathrm{wt} \% \mathrm{AIBN})$, HEMMA2 (1.0 wt $\%$ AIBN), HEMMA3 (1.5 wt $\%$ AIBN) and HEMMA4 (2.0 wt $\%$ AIBN). The schematic representation of this reaction is presented in Figure 1.

\subsection{Characterization of the HEA/MMA copolymers}

The characterization was performed on samples that were obtained after a reaction time of five hours. We are discussing the results below from these samples. As mentioned earlier, the samples were initially purified (only to these analyses) before recording infrared, hydroxyl value, NMR, and DSC analyses, and the determination of the HEA homopolymer and

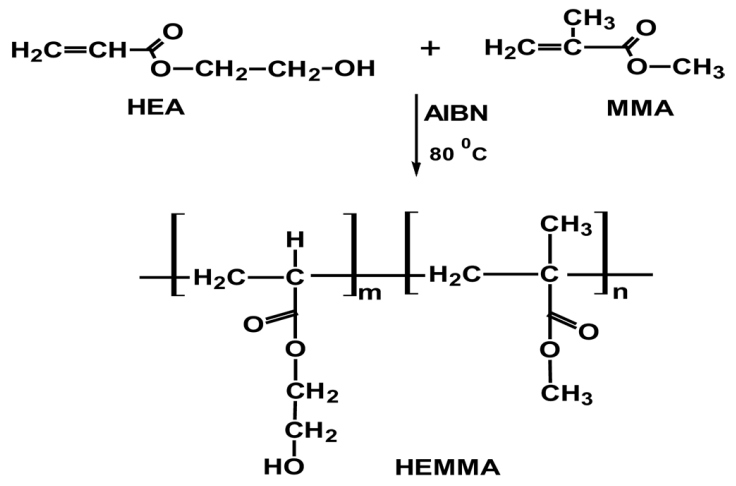

Figure 1. A schematic representation of the HEMMA copolymer synthesis.
HEMMA copolymers content. In order to acquire the data, approximately $10 \mathrm{~g}$ of samples were precipitated in hot xylene (MMA homopolymer is soluble, but the HEMMA copolymer is insoluble, furthermore in this solvent will be the residual HEA monomer), filtered and dried in an oven at $50{ }^{\circ} \mathrm{C}$ for $12 \mathrm{~h}$ under vacuum and finally weighted. Furthermore, the samples were subjected to soxhlet extraction with acetone for $12 \mathrm{~h}$ (extraction of HEA homopolymer) followed by drying in an oven at $50{ }^{\circ} \mathrm{C}$ for $12 \mathrm{~h}$ under vacuum, and weighted. The HEA homopolymer amount was determined employing initial weight of the sample, weight of the dry sample after of the precipitation process in hot xylene. The initial and final weight of the samples after soxhlet extraction process in acetone was used to determine the amount of HEMMA copolymers in the samples.

The HEMMA copolymers was re-precipitated in xylene and used to obtain MMA homopolymer. The solution of xylene (hypothetically containing the residual MMA monomer, residual HEA monomer and MMA homopolymer) was added to acetone and subjected to filtration process to obtain the MMA homopolymer.

Infrared analysis was carried out using a Perkin Elmer equipment model Spectrum One. The spectra were recorded with 8 scans, using resolution of $4 \mathrm{~cm}^{-1}$. The hydroxyl value analysis was made according to AOCS Cd 13-60. The dynamic light scattering analysis (DLS) was done using a Horiba equipment and at an angle of $90^{\circ}$, by using solutions of the samples in DMF (1 wt\%). The ${ }^{1} \mathrm{H}$ NMR analysis was carried out using a Bruker AC $300 \mathrm{MHz}$ spectrometer using dimethyl sulfoxide as the solvent. For gel permeation chromatography (GPC) analysis, the samples were dissolved in tetrahydrofuran and the analysis was performed in Waters HPLC equipment with millennium 2000 software for data acquisition and using polystyrene standards for the quantification. DSC analysis was carried out employing a TA Instrument model Q100 equipment, equipped with the refrigerated cooling system and using a heating and cooling rate of $30{ }^{\circ} \mathrm{C} / \mathrm{min}$ under nitrogen atmosphere. The decomposition temperature of the samples was determined by thermogravimetric analysis (TGA) using a TA instrument model Q500 equipment at a heating rate of $10{ }^{\circ} \mathrm{C} / \mathrm{min}$. The rheological measurements in solution were performed in a Bohling HRNano rotational rheometer (TA instruments) at a strain of $2 \%$ using concentric cylinder geometry. Studies of the HEMMA copolymers film properties (gloss, flexibility, and adherence) were performed according to methodologies reported in previous studies ${ }^{[22-24]}$.

\section{Results and Discussion}

The results from the gravimetric analysis are shown in Figure 2. Figure 2a, displays the conversion percentage of the reactions while Figure $2 b$, shows HEA homopolymers content in the HEMMA copolymers and finally Figure 2c, exhibit the yield of the HEAMMA copolymers, when only these were taken into account. The reaction conversion (Figure 2a) after $5 \mathrm{~h}$ was higher than $94 \%$, which increases with the initiator content in the synthesis. It is because the amount of initiator in the reaction mixture decreases the possibility of residual monomer by promoting them in polymerization process. Since no sample was purified initially before this 
analysis, it can be inferred that the unreacted amount of MMA and HEA was low and the system exhibited a high conversion percentage. This behavior has been observed in the free radical polymerization of $\mathrm{HEA}^{[25]}$.

It was apparent that the HEA homopolymer percentage in the samples (Figure 2b), increased with the amount of initiator. This result meant that the initiator concentration favored the formation of HEA homopolymer, possibly this was due to: a) there is high chemistry affinity between free radicals and HEA, b) high amount of HEA employed with respect that of the MMA, c) there is a high affinity between DMF and HEA, since both are highly hydrophilic (it improve the diffusion of species hydrophilic through solvent) and MMA is hydrophobic.

The gravimetric analysis did not display the presence of MMA homopolymer, suggesting that MMA homopolymer was not formed. Using the difference between conversions percentage of the reaction and the HEA homopolymer percentage in the HEMMA copolymers, was calculated, the conversion percentage (when only the HEMMA copolymers were taken into account) (Figure 2c). It can be visualized that the conversion percentages exhibited a trend with the initiator amount, however, the relation was not statistically significant (Figure 2c).

Figure 3 shows the IR spectra of the MMA and HEA (Figure 3a) and the purified HEMMA copolymers (Figure 3b). In the MMA spectrum (Figure 3a) a signal at $1439 \mathrm{~cm}^{-1}$ was observed due to bending of $-\mathrm{CH}_{3}$ groups. In the spectrum of the HEA (Figure 3a), the signal at $3416 \mathrm{~cm}^{-1}$ corresponds to stretching of $\mathrm{C}-\mathrm{OH}$ bonds. The signal that appears at $1725 \mathrm{~cm}^{-1}$ was due to absorption of the carbonyl groups $(-\mathrm{C}=\mathrm{O})$, and it appears in MMA and HEA spectra. The signal at $1638 \mathrm{~cm}^{-1}$ was due to stretching of $-\mathrm{CH}=\mathrm{CH}$ - bonds and that at $1410 \mathrm{~cm}^{-1}$, corresponds to the bending of $-\mathrm{CH}_{2}$.
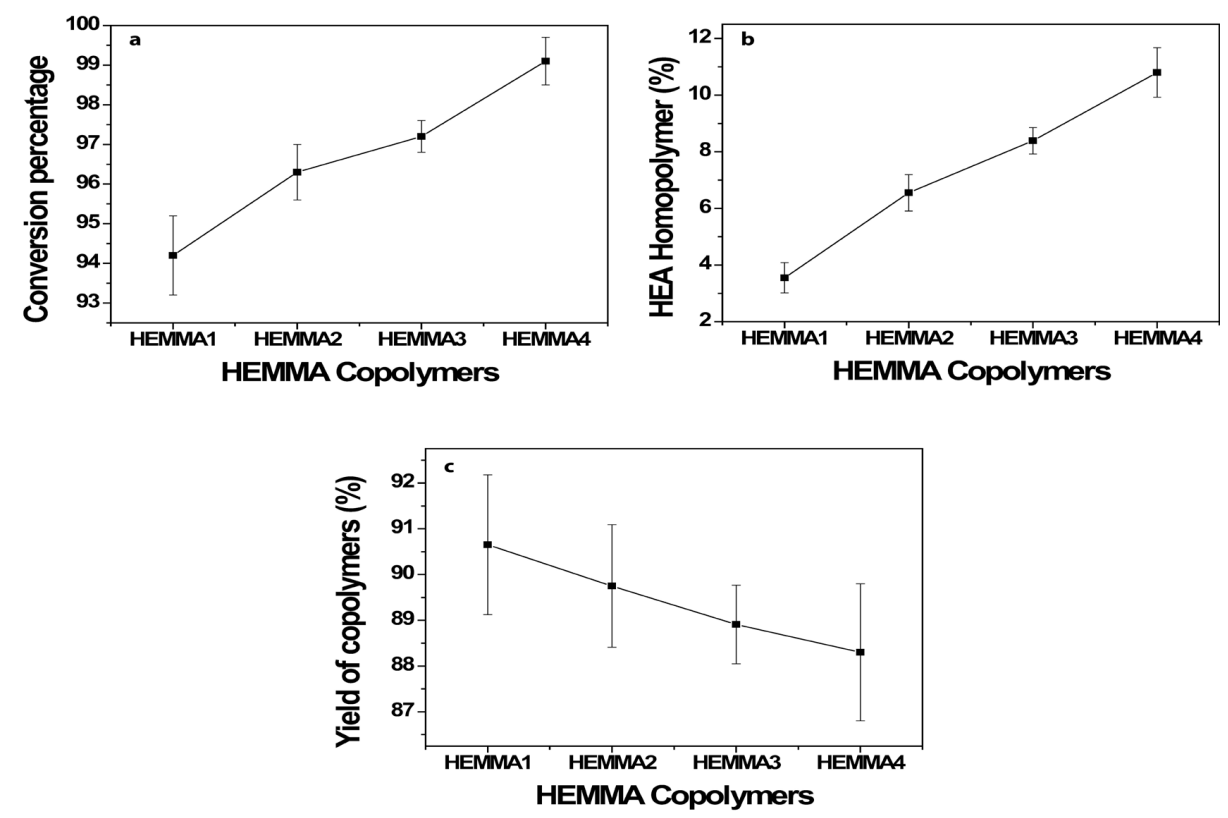

Figure 2. (a) Conversion percentage of the reactions and (b) HEA homopolymer percentage in the HEMMA copolymers and (c) yield of copolymers.
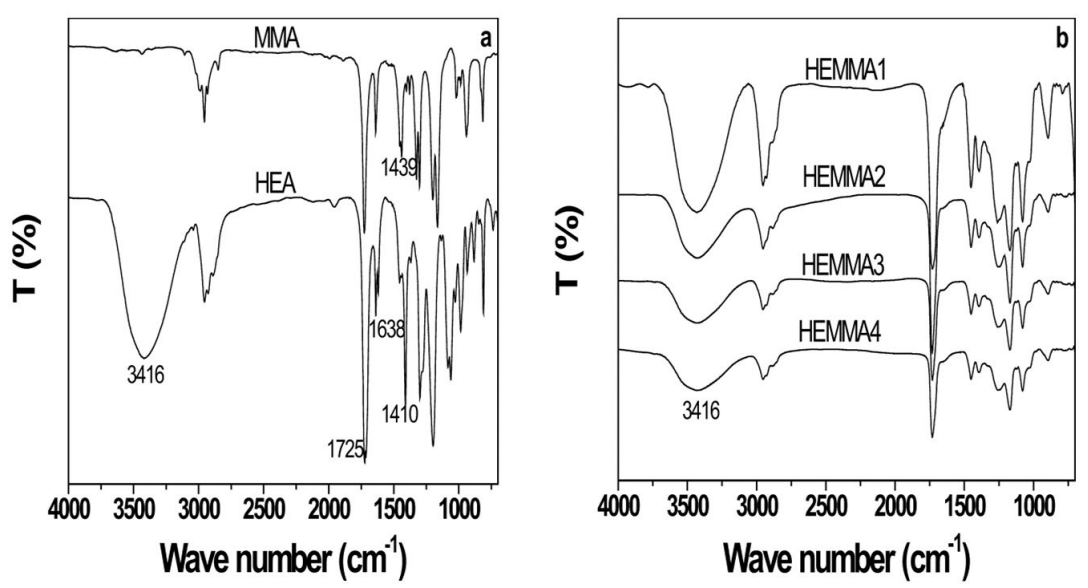

Figure 3. IR spectra (a) MMA and HEA and (b) HEMMA copolymers. 
Figure $3 \mathrm{~b}$ shows the IR spectra of the HEMMA copolymers. All samples showed a signal at $3416 \mathrm{~cm}^{-1}$ (C-OH bond) that decreased with the amount of AIBN and can be related to a reduction of HEA units in the copolymers, which is in good agreement with the results obtained to HEA homopolymer percentage in the copolymers (Figure 2b). This result is an indication that the repetitive units of the HEA and MMA were different in the HEMMA copolymers. None HEMMA copolymer exhibited the signal at $1638 \mathrm{~cm}^{-1}$ (stretching of $-\mathrm{CH}=\mathrm{CH}-$ ), which indicates that all monomers reacted, or the residual monomer was extracted during the purification process of the HEMMA copolymers.

The signals that appear at 1439 and $1410 \mathrm{~cm}^{-1}$ were due to $-\mathrm{CH}_{3}(\mathrm{MMA})$ and $-\mathrm{CH}_{2}$ (HEA) groups respectively. At $3416 \mathrm{~cm}^{-1}$ appears the signal for the $\mathrm{OH}$ groups. The presence of these signals is an evidence of the formation of the HEMMA copolymers. Considering the results obtained from IR, the HEA homopolymer amount increased from HEMMA1 to HEMMA4. These outcomes are in good agreement with the results obtained from gravimetric analysis (conversion percentage when only the HEMMA copolymers were taken into account).

The spectra of protonic nuclear magnetic resonance $\left({ }^{1} \mathrm{H}\right.$ NMR) of the HEMMA4 copolymer is presented in Figure 4. The signal around $1 \mathrm{ppm}$ is attributed to $-\mathrm{CH}_{3}$ protons. The $-\mathrm{CH}$ and $-\mathrm{CH}_{2}$ backbone protons resonated at 2.3 and between $1.5-1.9$ respectively ${ }^{[19]}$. The signal at $2.5 \mathrm{ppm}$ is attributed to the DMSO protons. Between 2.6 and $2.9 \mathrm{ppm}$ appear two signals, which correspond to protons of $\mathrm{CH}_{2} \mathrm{O}-$, which are originated of the etherification reaction between units of HEA in the HEMM4 copolymer; this is favored by the temperature [18], this signal have been observed by others authors in copolymerization reactions of $\mathrm{HEA}^{[18]}$, which argue that for every two losses in $(-\mathrm{OH})$ functional groups, there is a formation of two - $\mathrm{OCH} 2$ groups.

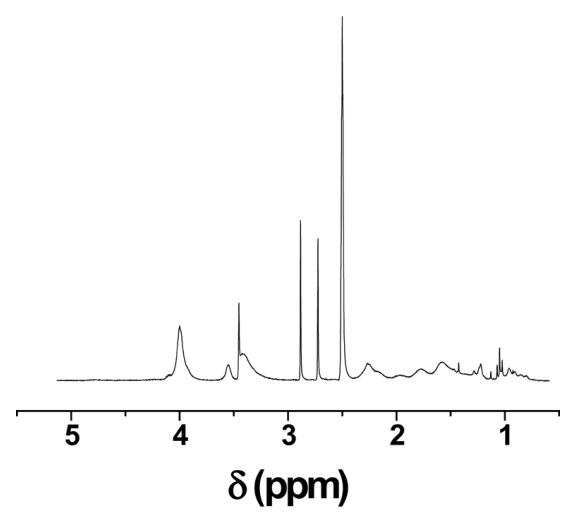

Figure 4. ${ }^{1} \mathrm{H}$ NMR of the HEMMA4 copolymer.
The signals at $3.4 \mathrm{ppm}$ and $3.6 \mathrm{ppm}$ corresponded to protons of the $-\mathrm{OCH}_{3}$ groups and methylene protons join to $\mathrm{OH}$ groups $\left(\mathrm{HO}_{-} \mathrm{CH}_{2}-\right)$ respectively ${ }^{[26]}$. At $4.1 \mathrm{ppm}$ appear the signals of the methylene protons of $-\mathrm{CH}_{2} \mathrm{O}$ - which are different that those that experimented etherification reactions ${ }^{[26]}$. The same signal has been observed by others authors ${ }^{[19,26]}$. The spectra did not exhibit signals due to protons $-\mathrm{CH}=\mathrm{CH}-(5.3 \mathrm{ppm})$, this is evidence that the monomers reacted. The presence of different signals indicated that the HEMMA copolymers were formed.

Table 1 , shows the respective values of number average molar mass (Mn), weight average molar mass (Mw) and the polydispersity index (PI) of the HEMMA copolymers. The behavior showed by the HEMMA copolymers with the increase in the AIBN content was expected since with it, the molar mass would be reduced, due to a high number of termination reactions between macroradicals. The lowest $\mathrm{Mn}$ and $\mathrm{Mw}$ values were due to high amounts of AIBN and polymerization method employed in the synthesis. The variation in polydispersity was not significant. The Mn values decreased with the amount of HEA homopolymer formed, but $\mathrm{Mw}$ did not follow the same trend, which is possibly due to crosslinking reaction exhibited by this material as it was demonstrated by NMR analysis.

The VOH of the HEMMA copolymers (Table 1), decreased with increase in AIBN content employed in the synthesis. This result is in agreement with that obtained with the HEA homopolymer content, since it was expected that when the content of HEA homopolymer is high the amount of the units of HEA in the copolymers will be low.

The results of VOH are in accordance with the results presented by IR analysis. Castor oil with a VOH of $164 \mathrm{mg}$ $\mathrm{KOH} / \mathrm{g}$ sample has been employed to obtain acrylic ester by esterification reaction between $\mathrm{OH}$ groups (castor oil) and acid groups of acrylic acid ${ }^{[27]}$. In the future, the $\mathrm{VOH}$ obtained to the HEMMA copolymers, will allow that these materials be modified with fatty acids to obtain alkyd resins or others hybrid materials.

Figure 5 presents the volume (Figure $5 \mathrm{a}$ ) and intensity (Figure $5 b$ ) size distributions of the HEMMA copolymers. The hydrodynamic dimensions (volume) of the copolymers (Figure 5a) were nanometric as follows: HEMMA1: 18, HEMMA2: 23, HEMMA3: 15 and HEMMA4: $13.0 \mathrm{~nm}$. According to the results of GPC, it was expected that the HEMMA1 copolymer would exhibit the higher hydrodynamic dimensions, but this was not the behavior. This was possibly due to HEMMA copolymers exhibiting different processes such as aggregations through interactions between $\mathrm{OH}$ groups (hydrogen bonds) or crosslinking reactions (through HEA). All volume size distributions were monodisperse and nanometric.

Table 1. Mn, Mw, PI and VOH values of the HEMMA copolymers.

\begin{tabular}{ccccc}
\hline HEMMA Copolymers & Mn (g/mol) & Mw (g/mol) & PI & VOH (mg KOH/g sample) \\
\hline HEMMA1 & 7620 & 9486 & 1.2 & $130 \pm 2.0$ \\
HEMMA2 & 7073 & 10457 & 1.5 & $111 \pm 2.0$ \\
HEMMA3 & 6446 & 7579 & 1.2 & $100 \pm 1.0$ \\
HEMMA4 & 5619 & 7744 & 1.4 & $82 \pm 2.0$ \\
\hline
\end{tabular}



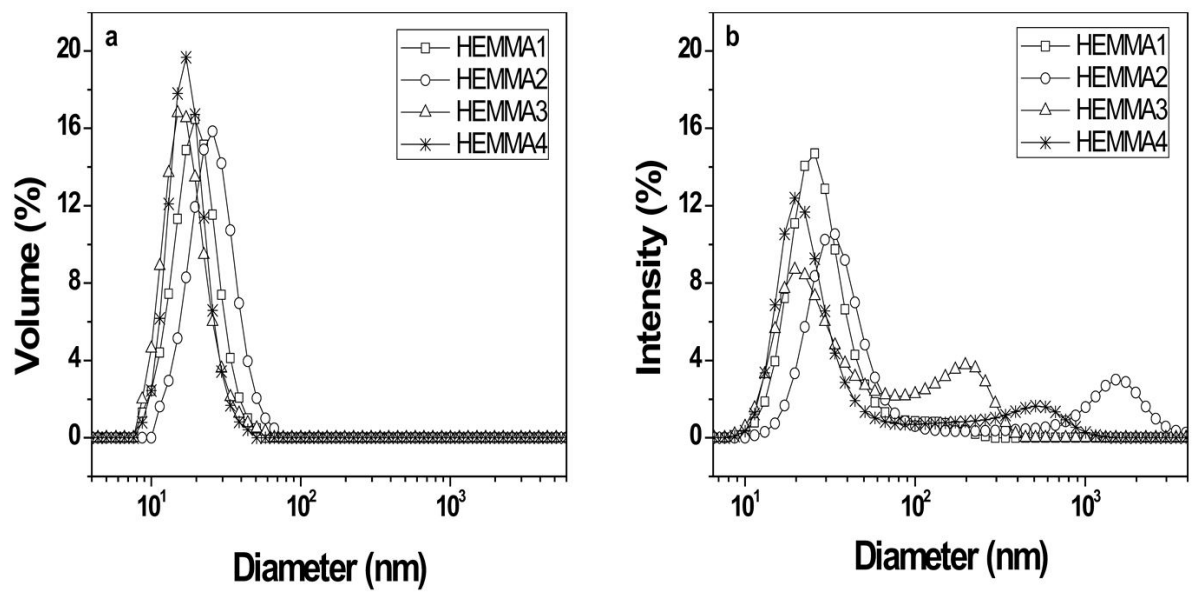

Figure 5. Size distributions of the HEMMA copolymers (a) Volume and (b) Intensity.

The intensity size distributions (Figure $5 \mathrm{~b}$ ) present other distributions with diameters (d) higher than $100 \mathrm{~nm}$ for the HEMMA2, HEMMA3 and HEMMA4. This is an indication of the presence of aggregates. The intensity distribution is very sensible for detecting the presence of aggregates because large particles scatter more light than small ones. The nanometric hydrodynamic dimensions obtained in this study were mainly nanometric due to the high amounts of AIBN and polymerization method employed in the synthesis. This result was very important because these materials exhibit high structural packing. The presence of aggregations in the samples HEMMA2, HEMMA3, and HEMMA4, may be attributed mainly to the interactions between $\mathrm{OH}$ groups (hydrogen bonds) originated from HEA homopolymers. These copolymers exhibited higher HEA homopolymer content than that of the HEMMA1 copolymer (not exhibited aggregation). The interactions through $\mathrm{OH}$ groups has been reported by some authors to yield hyperbranched polyester polyols by employing good solvents such as DMF and $\mathrm{DMSO}^{[28-31]}$. Since the aggregations appear in intensity size distribution but it does not appear in volume size distribution, it can be inferred that its number is lowest.

Figure 6 shows the rheological behavior of the HEMMA copolymers in solution (in each case the solid content was $50 \mathrm{wt} \%$ ). The HEMMA copolymer viscosities at $10 \mathrm{~s}^{-1}$ were as follows: HEMMA1: 7.4 Pa.s, HEMMA2: 6.0 Pa.s, HEMMA3: 5.0 Pa.s and HEMMA4: 1.7 Pa.s. The low viscosity of the HEMMA4 may be due to low crosslinking and entanglement degrees. The viscosity decreased with the increasing of the AIBN content employed in the synthesis, and this result was expected because in this same sense occurs a reduction in molar mass. All viscosity values of the HEMMA were low due to low molar mass. All copolymers presented a stable region in viscosity, but this disappeared at shear rates higher than $50 \mathrm{~s}^{-1}$, the fluids hence became shear thinning and this behavior was attributed to disentanglement of the polymer chains, but also to dissociation of interactions. The same behavior has been observed in hydroxyethyl methacrylatebutyl acrylate copolymers ${ }^{[32]}$.

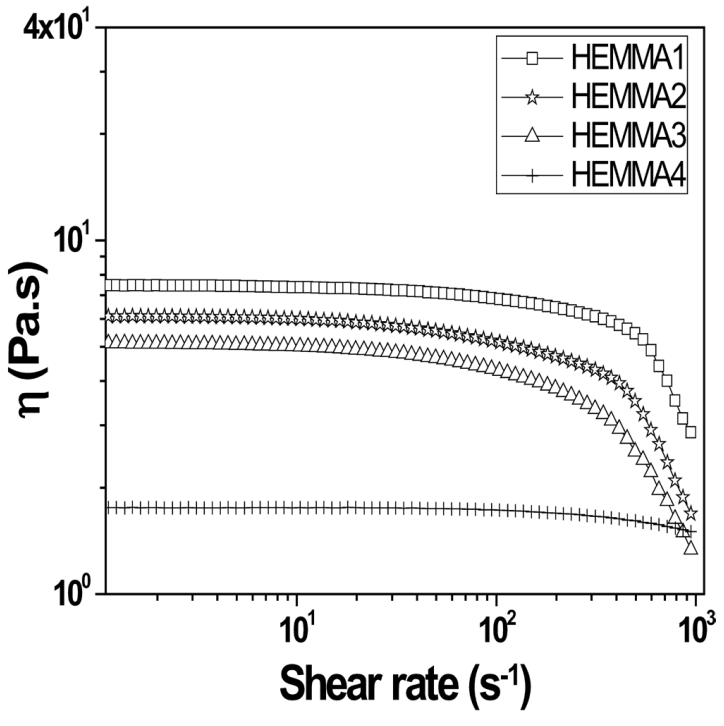

Figure 6. Rheological behavior of the HEMMA copolymers.

Since the rheological behavior of the HEMMA copolymers was consistent with the molar mass, it was difficult to identify the influence of the crosslinking process on the rheological behavior of these materials, because this process is not reversible.

According to results obtained by DLS analysis (intensity size distribution), it apparent that only HEMMA2, HEMMA3 and HEMMA4 copolymers exhibited a pseudoplastic behavior due to dissociation of interactions. Moreover, by DLS analysis it was evidenced that these copolymers exhibited aggregation. However, HEMMA1 copolymer (Figure $5 b$ ), did not exhibit aggregations but showed pseudoplastic behavior, due to disentanglement of the chains. The HEMMA4 copolymer exhibited lower pseudoplastic behavior (reduction in viscosity with the increasing of shear rate) due to lowest molecular weight and reduction in the possibility of entanglement. 
Figure 7 presents the results of the thermal analysis. Figure 7a shows the thermograms obtained by differential scanning calorimetry (DSC) and Figure $7 \mathrm{~b}$ the thermograms obtained by thermogravimetric analysis (TGA). All glass transition temperatures $\left(\mathrm{T}_{\mathrm{g}}\right)$ of the HEMMA copolymers (Figure 7a) were different to values of PHEA $\left(-15^{\circ} \mathrm{C}\right)^{[8]}$ and MMA homopolymer $\left(105^{\circ} \mathrm{C}\right)^{[11]}$. This is evidence that the HEMMA copolymers were formed. The $\mathrm{T}_{\mathrm{g}}$ values of the HEMMA copolymers were as follows: HEMMA1: $32^{\circ} \mathrm{C}$, HEMMA2: $35^{\circ} \mathrm{C}$, HEMMA3: $26^{\circ} \mathrm{C}$ and HEMMA4: $33^{\circ} \mathrm{C}$.

The results of DSC analysis do not correspond with the molar mass and $\mathrm{VOH}$ and this may be due to crosslinking reactions through HEA in the copolymers (Figure 5), interaction between $\mathrm{OH}$ groups and different compositions of the copolymers since the MMA is rigid, and HEA is elastic $^{[8,11]}$.

With the increasing on molar mass, there was a reduction in free volume and chain mobility, therefore it was expected that $T_{g}$ values increased with the molecular weight, but none HEMMA copolymers did not exhibit this behavior. Furthermore, it was expected that the $\mathrm{T}_{\mathrm{g}}$ values would also increase with the $\mathrm{VOH}$, since these values augmented with the $\mathrm{OH}$ group numbers in the chain and the probability of occurrence of interactions through the $\mathrm{OH}$ groups was higher than when the $\mathrm{OH}$ group numbers were low. It can be concluded that the interactions degree between $\mathrm{OH}$ groups and crosslinking degree were different for every HEMMA copolymer.

It is apparent that the amount of HEA homopolymer in the HEMMA4 is highest. Also, the number of MMA units in this copolymer was higher than those of the others copolymers. It is possible that HEMMA4 copolymer contains lowest number of HEA units in its structure long with low Mn value yet displaying $\mathrm{T}_{\mathrm{g}}$ similar to HEMMA1 and HEMMA2. This result suggests that the distribution and the number of unit of HEA and MMA in HEMMA copolymers were different.
The crosslinking reaction of HEA has been reported ${ }^{[33]}$. In a previous study, it was reported that the curing rate for HEA was faster than 2-ethylhexyl acrylate, 2-hydroxyethyl methacrylate, and hydroxypropyl acrylate and hydroxypropyl methacrylate ${ }^{[33]}$.

All copolymers exhibited a weight loss between 200 and $350{ }^{\circ} \mathrm{C}$ (Figure $7 \mathrm{~b}$ ), which corresponds to oligomers and copolymers of low molecular weight. Likewise, at approximately $400{ }^{\circ} \mathrm{C}$ a great weight loss for all HEMMA copolymers appears. The temperatures of thermal decomposition of the HEMMA copolymers were as follows: HEMMA1: $414{ }^{\circ} \mathrm{C}$, HEMMA2: $416{ }^{\circ} \mathrm{C}$, HEMMA3: $423{ }^{\circ} \mathrm{C}$ and HEMMA4: $418{ }^{\circ} \mathrm{C}$. There is no correlation with the molecular weight, initiator amount, HEA homopolymer amount or $\mathrm{VOH}$. Therefore, this behavior is possibly attributed to a different number of interactions, homopolymerizations, and crosslinking reactions.

Table 2 presents the results of adhesion, flexibility and gloss analyses of the HEMMA copolymers. The adhesion and flexibility of the copolymers were good. It has been demonstrated that acrylic polymers exhibit good adhesion ${ }^{[2,34]}$. In the case of flexibility, this result was expected because the proportion of HEA employed in the synthesis, was high with respect to MMA.

The gloss values were very similar to the HEMMA copolymers. Therefore, the behavior shown by the HEMMA copolymers in gloss was possibly due to small variations in refractive index and roughness on the surfaces.

Table 2. Values of gloss, adhesion, and flexibility of the HEMMA copolymers.

\begin{tabular}{cccc}
\hline $\begin{array}{c}\text { HEMMA } \\
\text { Copolymers }\end{array}$ & Gloss $\left(\mathbf{2 0}^{\circ}\right)$ & Adhesion (\%) & Flexibility \\
\hline HEMMA1 & 132 & 100 & Pass \\
HEMMA2 & 132 & 100 & Pass \\
HEMMA3 & 137 & 100 & Pass \\
HEMMA4 & 126 & 100 & Pass \\
\hline
\end{tabular}

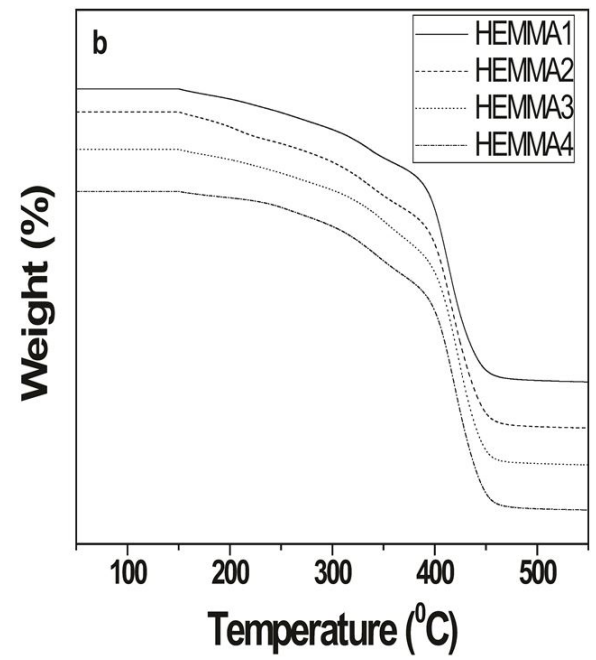

Figure 7. Thermograms of the HEMMA copolymers (a) DSC and (b) TGA. 


\section{Conclusions}

This study made an important contribution to the synthesis of functional copolymers since HEMMA copolymers were obtained, and they may be an alternative to the synthesis of new materials. With the AIBN content, decreased the amount of HEA in the copolymers, which is attributed homopolymerization of HEA. The initiator concentration improves the conversion percentage of the reaction (take into account HEA homopolymer and HEMMA copolymer formed) and the formation of HEA homopolymer, but it did not improve the formation of HEMMA copolymers. The molar mass and the viscosity of the copolymers decreased with the amount of AIBN. The VOH of the copolymers was between 82 and $130 \mathrm{mg} \mathrm{KOH} / \mathrm{g}$ sample and their hydrodynamic dimensions were nanometric. They presented an adequate $\mathrm{VOH}$, which possibly would allow esterification reactions between $\mathrm{OH}$ groups of the HEMMA copolymers and $\mathrm{COOH}$ groups of fatty acids (preparation of alkyd resins type comb). The viscosity of the copolymers was lower than 10 Pa.s. The copolymers obtained were amorphous and this may explain the good flexibility that they exhibited.

\section{Acknowledgements}

We would like to thank The Comité Para el Desarrollo de la Investigación (CODI) de la Universidad de Antioquia (Colombia) for their financial support of this investigation.

\section{References}

1. Murillo, E. A., López, B. L., \& Hess, M.(2004). Synthesis and characterization of a pressure-sensitive adhesive based on an isobutyl acrylate / 2-ethylhexyl acrylate copolymer. e-polymer, 28, 1-10.

2. Silvestri, D., Gagliardi, M., Cristallini, C., Barbani, N., \& Giusti, P. (2009). Different composition poly (methyl methacrylateco-butyl methacrylate) copolymers through seeded semi-batch emulsion polymerization. Polymer Bulletin, 63(3), 423-439. http://dx.doi.org/10.1007/s00289-009-0095-2.

3. Yeon, K., Yeon, J., Choi, Y., \& Min, S. (2014). Deformation behavior of acrylic polymer concrete: Effects of methacrylic acid and curing temperature. Construction \& Building Materials, 63, 125-131. http://dx.doi.org/10.1016/j.conbuildmat.2014.04.051.

4. Chen, J. J., Ahmad, A. L., \& Ooi, B. S. (2014). Thermoresponsive properties of poly(N-isopropylacrylamide-coacrylic acid) hydrogel and its effect on copper ion removal and fouling of polymer-enhanced ultrafiltration. Journal of Membrane Science, 469, 73-86. http://dx.doi.org/10.1016/j. memsci.2014.05.062.

5. Murillo, E. A., \& López, B. L. (2011). Novel waterborne hyperbranched acrylated-maleinized alkyd resins. Progress in Organic Coatings, 72(4), 731-738. http://dx.doi.org/10.1016/j. porgcoat.2011.08.004.

6. Miller, K. R., \& Soucek, M. D. (2012). Photopolymerization of biocompatible films containing poly(lactic acid). European Polymer Journal, 48(12), 2107-2116. http://dx.doi.org/10.1016/j. eurpolymj.2012.08.006.

7. Çaykara, T., Özyürek, C., Kantoğlu, Ö., \& Erdoğan, B. (2003). Thermal behavior of poly(2-hydroxyethyl methacrylate-maleic acid) networks. Polymer Degradation \& Stability, 80(2), 339343. http://dx.doi.org/10.1016/S0141-3910(03)00018-1.

8. Taghizadeh, S. M., \& Ghasemi, D. (2010). Synthesis and optimization of a four-component acrylic-based copolymer as pressure sensitive adhesive. Iranian Polymer Journal, 19, 343-352.

9. Kowalczyk, K., Spychaj, T., Ubowska, A., \& Schmidt, B. (2014). Industrially applicable methods of poly(methyl methacrylate)/organophilic montmorillonite nanocomposites preparation: processes and cast materials characterization. Applied Clay Science, 97, 96-103. http://dx.doi.org/10.1016/j. clay.2014.05.011.

10. Ozbay, S., \& Erbil, H. Y. (2014). Solution copolymerization of perfluoroalkyl ethyl methacrylate with methyl methacrylate and butyl acrylate: synthesis and surface properties. Colloids and Surfaces. A, Physicochemical and Engineering Aspects, 452, 9-17. http://dx.doi.org/10.1016/j.colsurfa.2014.03.054.

11. Shan, X., Liu, Y. C., \& Lam, Y. C. (2008). Studies of polymer deformation and recovery in micro hot embossing. Microsystem Technologies, 14(7), 1055-1060. http://dx.doi.org/10.1007/ s00542-007-0486-y.

12. Moayed, S. H., Fatemi, S., \& Pourmahdian, S. (2007). Synthesis of a latex with bimodal particle size distribution for coating applications using acrylic monomers. Progress in Organic Coatings, 60(4), 312-319. http://dx.doi.org/10.1016/j. porgcoat.2007.07.023.

13. Park, S. G., Kwak, N. S., Hwang, C. W., Park, H., \& Hwang, T. S. (2012). Synthesis and characteristics of aminated vinylbenzyl chloride-co-styrene-co-hydroxyethyl acrylate anion-exchange membrane for redox flow battery applications. Journal of Membrane Science, 423, 429-437. http://dx.doi.org/10.1016/j. memsci.2012.08.040.

14. Dziczkowski, J., \& Soucek, M. D. (2012). Factors influencing the stability and film properties of acrylic/alkyd water-reducible hybrid systems using a response surface technique. Progress in Organic Coatings, 73(4), 330-343. http://dx.doi.org/10.1016/j. porgcoat.2011.03.005.

15. Harakawa, H., Kasari, A., Tominaga, A., \& Yabuta, M. (1998). The rheological properties of an aqueous acrylic dispersion suitable for automotive waterborne basecoats. Progress in Organic Coatings, 34(1-4), 84-90. http://dx.doi.org/10.1016/ S0300-9440(98)00003-4.

16. Gómez, J. L., Monleón, M., Gallego, G., Peidro, N., Pérez, V., Pissis, P., \& Kyritsis, A. (1999). Poly(methyl acrylate)/ poly(hydroxyethyl acrylate) sequential interpenetrating polymer networks. Miscibility and water sorption behavior. Journal of Polymer Science. Part B, Polymer Physics, 37, 1587-1599. http:// dx.doi.org/10.1002/(SICI)1099-0488(19990715)37:14<1587::AIDPOLB4>3.0.CO;2-U.

17. Pourjavadi, A., Samadi, M., \& Ghasemzadeh, H. (2008). Fastswelling superabsorbent hydrogels from poly(2-hydroxy ethyl acrylate-co-sodium acrylate) grafted on starch. Stärke, 60(2), 79-86. http://dx.doi.org/10.1002/star.200700666.

18. Sahloul, N., Emwas, A., Power, W., \& Penlidis, A. (2005). Ethyl acrylate $\square$ hydroxyethyl acrylate and hydroxyethyl acrylate $\square$ methacrylic acid: reactivity ratio estimation from cross $\square$ linked polymer using high resolution magic angle spinning spectroscopy. Journal of Macromolecular Science, Part A: Pure and Applied Chemistry, 42(10), 1369-1385. http:// dx.doi.org/10.1080/10601320500205558.

19. Mun, G. A., Nurkeeva, Z. S., Dergunov, S. A., Nam, I. K., Maimakov, T. P., Shaikhutdinov, E. M., Lee, S. C., \& Park, K. (2008). Studies on graft copolymerization of 2-hydroxyethyl acrylate onto chitosan. Reactive \& Functional Polymers, 68(1), 389-395. http://dx.doi.org/10.1016/j.reactfunctpolym.2007.07.012.

20. Lee, J. H., \& Kim, J. J. (2014). Poly(hydroxyethyl acrylate-cocoumaryl acrylate) as a photo-responsive amphiphile. Journal of Industrial and Engineering Chemistry, 20(5), 3075-3080. http://dx.doi.org/10.1016/j.jiec.2013.11.046. 
21. Henini, G., Souahi, F., \& Laidani, Y. (2012). Tracking offline conversion solution polymerization of methyl methacrylate / vinyl acetate in toluene in a reactor calorimeter. Procedia Engineering, 33, 225-233. http://dx.doi.org/10.1016/j. proeng.2012.01.1198.

22. Murillo, E. A., Vallejo, P. P., \& López, B. L. (2010). Synthesis and characterization of hyperbranched alkyd resins based on tall oil fatty acids. Progress in Organic Coatings, 69(3), 235240. http://dx.doi.org/10.1016/j.porgcoat.2010.04.018.

23. Murillo, E. A., Vallejo, P. P., \& López, B. L. (2011). Effect of tall oil fatty acids content on the properties of novel hyperbranched alkyd resins. Journal of Applied Polymer Science, 112(6), 3151-3158. http://dx.doi.org/10.1002/app.33502.

24. Murillo, E. A., López, B. L., \& Brostow, W. (2011). Synthesis and characterization of novel alkyd-silicone hyperbranched nanoresins with high solid contents. Progress in Organic Coatings, 72(3), 292-298. http://dx.doi.org/10.1016/j. porgcoat.2011.04.019.

25. Chen, S., Hu, T., Tian, Y., Chen, L., \& Pojman, J. A. (2007). Facile synthesis of poly(hydroxyethyl acrylate) by frontal freeradical polymerization. Journal of Polymer Science. Part A, Polymer Chemistry, 45(5), 873-881. http://dx.doi.org/10.1002/ pola.21865.

26. Moad, G., Rizzardo, E., \& Thang, S. H. (2005). Living radical polymerization by the RAFT process. Australian Journal of Chemistry, 58(6), 379-410. http://dx.doi.org/10.1071/CH05072.

27. Jain, M., Vora, R. A., \& Satpathy, U. S. (2003). Kinetics of emulsion copolymerization of methylmethacrylate and ethylacrylate: effect of type and concentration of initiator in unseeded polymerization system. European Polymer Journal, 39(10), 2069-2076. http://dx.doi.org/10.1016/S00143057(02)00347-6.

28. Xu, M., Yan, X., Cheng, R., \& Yu, X. (2001). Investigation into the solution properties of hyperbranched polymer. Polymer
International, 50(12), 1338-1345. http://dx.doi.org/10.1002/ pi.740.

29. Murillo, E. A., Vallejo, P. P., Sierra, L., \& López, B. L. (2009). Characterization of hyperbranched polyol polyesters based on 2,2-bis (methylol propionic acid) and pentaerythritol. Journal of Applied Polymer Science, 112(1), 200-207. http://dx.doi. org/10.1002/app.29397.

30. Murillo, E. A., Cardona, A., \& López, B. L. (2011). Rheological behavior in the molten state and solution of hyperbranched polyester of fourth and fifth generation. Journal of Applied Polymer Science, 119(2), 929-935. http://dx.doi.org/10.1002/ app.32774.

31. Murillo, E. A., Vallejo, P. P., \& López, B. L. (2010). Characterization of hydroxylated hyperbranched polyesters of fourth and fifth generation. Polymer, 10(1), 1347-1358. http://dx.doi.org/10.1515/epoly.2010.10.1.1347.

32. Perera, D., \& Shanks, R. A. (1995). Synthesis and rheological properties of soluble poly(hydroxyethyl methacrylate) and some copolymers. Polymer International, 36(4), 303-308. http://dx.doi.org/10.1002/pi.1995.210360401.

33. McManus, N. T., Kim, J. D., \& Penlidis, A. (1998). Observations on styrene-hydroxyethyl acrylate and styrene-hydroxyethyl acrylate-ethyl acrylate polymerizations. Polymer Bulletin, 41(6), 661-668. http://dx.doi.org/10.1007/s002890050415.

34. Peykova, Y., Lebedeva, O. V., Diethert, A., Müller-Buschbaum, P., \& Willenbacher, N. (2012). Adhesive properties of acrylate copolymers: effect of the nature of the substrate and copolymer functionality. International Journal of Adhesion and Adhesives, 34,107-116. http://dx.doi.org/10.1016/j.ijadhadh.2011.12.001.

Received: Aug. 28, 2014

Revised: Sept. 14, 2015

Accepted: May 16, 2016 